\title{
Pulmonary tuberculosis masquerading as metastatic lung disease
}

\begin{abstract}
A 30-year-old, HIV-negative female, who had never smoked, was referred for evaluation of lung metastasis. She had abdominal pain, occasional fever and weight loss for 3 months. Progressive breathlessness was present for 15 days. Her physical examination was unremarkable. Chest radiography (figure 1) showed bilateral nodular opacities with indistinct margins in all the lung zones. Computed tomography (CT) of the thorax demonstrated multiple round nodules of variable sizes scattered throughout both lung fields, suggestive of metastatic lung disease. Abdominal ultrasonography revealed multiple large retroperitoneal lymph nodes, which were confirmed on CT. Several sputum samples for acid-fast bacilli (AFB) and malignant cytology were negative. Her bronchial aspirate was positive for AFB and GeneXpert (Cepheid, Sunnyvale, CA, USA) detected Mycobacterium tuberculosis without rifampicin resistance. Transbronchial biopsy revealed caseating granulomas consistent with pulmonary tuberculosis. She was then referred to DOTS (directly observed therapy, short course) centre for initiation of antituberculous therapy. Subsequently, the bronchial aspirate also cultured M. tuberculosis.

The typical presentation of pulmonary tuberculosis is easily recognisable but the disease, a well-known masquerader, can be a source of diagnostic confusion radiologically when the presentation is atypical. The most common and feared cause of multiple round opacities of varying sizes in the lungs is metastasis. Pulmonary tuberculosis, as a cause of such a radiological presentation, is very rare, with only sporadic reports [1-3] documenting such an appearance. In an appropriate setting, pulmonary tuberculosis should be considered in the differential diagnosis of bilateral pulmonary nodules simulating metastasis. This could possibly prevent invasive interventions being performed to establish a diagnosis [2].
\end{abstract}
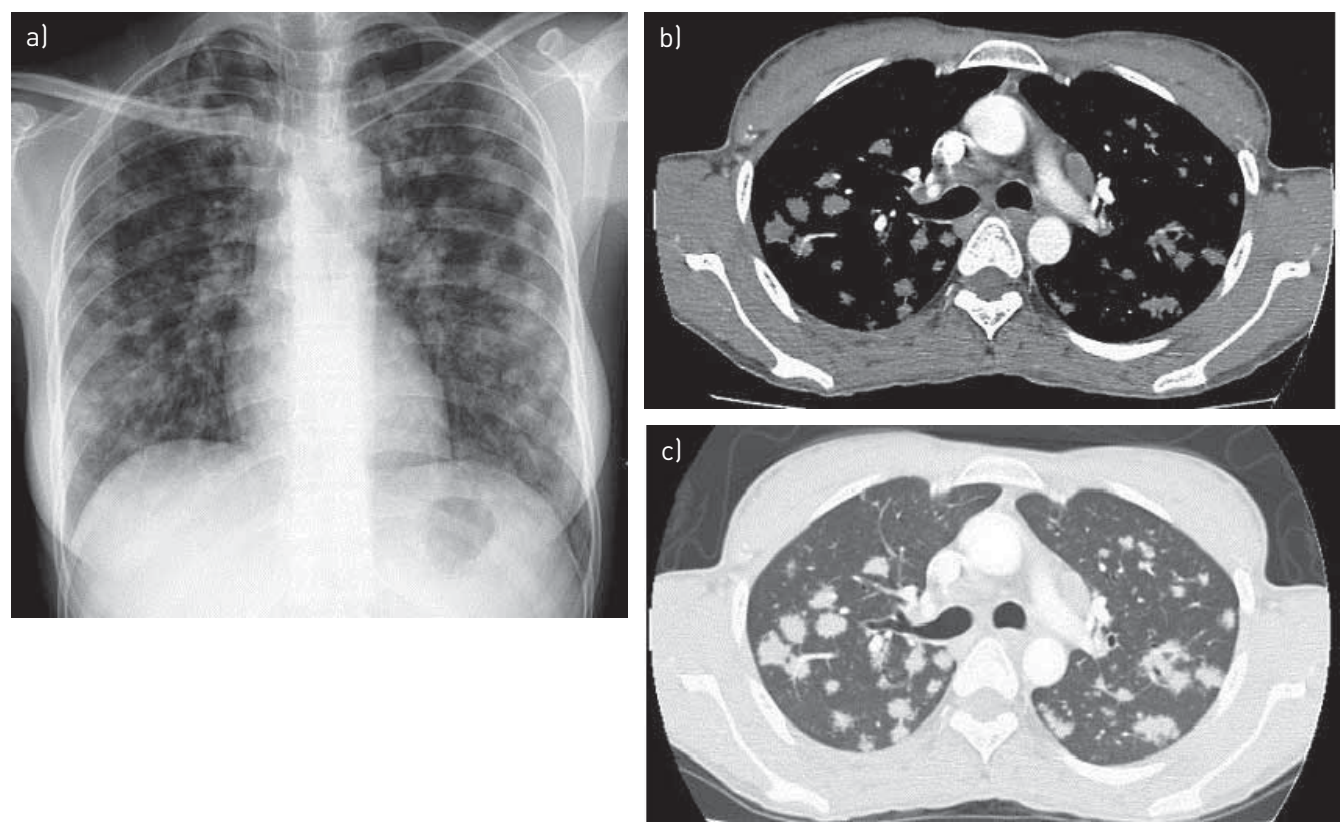

FIGURE 1 a) Chest radiography, performed on presentation, showed bilateral nodular opacities with indistinct margins in all the lung zones. $b, c)$ Computed tomography of the thorax demonstrated multiple round nodules of variable sizes scattered throughout both lung fields, suggestive of pulmonary metastasis. b) Mediastinal window and cl lung window. 
- $@ E R S p u b l i c a t i o n s$

Pulmonary TB should be considered in the differential diagnosis of bilateral pulmonary nodules simulating metastasis http://ow.ly/TLQl4

Vikas Pilaniya, Kamal Gera, Shekhar Kunal and Ashok Shah

Dept of Respiratory Medicine, Vallabhbhai Patel Chest Institute, University Of Delhi, Delhi, India.

Correspondence: Ashok Shah, Dept of Respiratory Medicine, Vallabhbhai Patel Chest Institute, University Of Delhi, PO Box 2101, Delhi 110 007, India. E-mail: ashokshah99@yahoo.com

Received: March 172015 | Accepted: March 192015

Conflict of interest: None declared.

Provenance: Submitted article, peer reviewed.

References

1 Ariyürek $\mathrm{MO}$, Karçaaltincaba $\mathrm{M}$, Demirkazik $\mathrm{FB}$, et al. Bilateral multiple pulmonary tuberculous nodules mimicking metastatic disease. Eur J Radiol 2002; 44: 33-36.

2 Paci M, Cavazza A, Ferrari G, et al. Pulmonary tuberculosis mimicking lung metastasis. J Thorac Oncol 2008; 3: 660-661.

3 Kant S, Saheer S, Prakash V, et al. Bilateral nodular pulmonary tuberculomas simulating metastatic disease. BMJ Case Rep 2011; DOI: 10.1136/bcr.11.2010.3539.

Eur Respir Rev 2016; 25:97-98 | DOI: 10.1183/16000617.00002315 | Copyright (C)ERS 2016. ERR articles are open access and distributed under the terms of the Creative Commons Attribution Non-Commercial Licence 4.0. 